\title{
Peripheral Ischemia
}

National Cancer Institute

\section{Source}

National Cancer Institute. Peripheral Ischemia. NCI Thesaurus. Code C78535.

Deficient blood distribution to the limbs caused by narrowing or obstruction of the lumen of the peripheral arteries. 